Delft University of Technology

\title{
NUFFT-Based Range Migration for 3-D Imaging with Irregular Planar Array
}

Wang, Jianping; Yarovoy, Alexander

DOI

10.1109/ICEAA.2019.8879356

Publication date

2019

Document Version

Final published version

Published in

2019 International Conference on Electromagnetics in Advanced Applications (ICEAA)

\section{Citation (APA)}

Wang, J., \& Yarovoy, A. (2019). NUFFT-Based Range Migration for 3-D Imaging with Irregular Planar Array. In 2019 International Conference on Electromagnetics in Advanced Applications (ICEAA): Proceedings (pp. 671-675). [8879356] IEEE . https://doi.org/10.1109/ICEAA.2019.8879356

\section{Important note}

To cite this publication, please use the final published version (if applicable).

Please check the document version above.

\section{Copyright}

Other than for strictly personal use, it is not permitted to download, forward or distribute the text or part of it, without the consent of the author(s) and/or copyright holder(s), unless the work is under an open content license such as Creative Commons.

\section{Takedown policy}

Please contact us and provide details if you believe this document breaches copyrights.

We will remove access to the work immediately and investigate your claim. 
Green Open Access added to TU Delft Institutional Repository 'You share, we take care!' - Taverne project

\section{https://www.openaccess.nl/en/you-share-we-take-care}

Otherwise as indicated in the copyright section: the publisher is the copyright holder of this work and the author uses the Dutch legislation to make this work public. 


\title{
NUFFT-Based Range Migration for 3-D Imaging with Irregular Planar Array
}

\author{
Jianping Wang, Alexander Yarovoy \\ Faculty of Electrical Engineering, Mathematics and Computer Science \\ Delft University of Technology \\ Delft, the Netherlands \\ \{J.Wang-4, A.Yarovoy\}@ tudelft.nl
}

\begin{abstract}
In this paper, a Nonuniform fast Fourier transform (NUFFT)-based range migration algorithm (RMA) is proposed for three-dimensional (3-D) imaging with irregular planar arrays. The proposed imaging algorithm takes advantage of NUFFT to reconstruct the signal spectra in the wavenumber domain, which overcomes the constraint of irregular spatial sampling on the utilization of Fourier-based imaging algorithms. Compared to the conventional coherent summation algorithms, i.e., backprojection and Kirchhoff migration, it is a very efficient method for 3-D image formation with nonuniform spatial sampling. To demonstrate its imaging performance, some numerical simulations are presented and discussed.

Index Terms-Nonuniform fast Fourier transform (NUFFT), planar array, nonuniform spatial sampling, range migration, three-dimensional(3-D) imaging.
\end{abstract}

\section{INTRODUCTION}

Planar array-based imaging systems have been used for various applications, such as through-the-wall imaging, concealed weapon detection, ground penetrating radar, and medical imaging, to list a few. To get high cross-range resolutions and high dynamic ranges, more and more imaging systems explore irregular/random array designs to suppress the shadowing effects, which leads to irregular or/and nonuniform spatial sampling of the signals [1]-[3]. Meanwhile, some new imaging configurations, for instance, 3-D imaging with rotating arrays, synthesize a large planar aperture by rotating a small antenna array around a point on the aperture plane [4]-[7]. The synthetic arrays naturally acquire signals on an irregular grid in space. In both cases, the irregular/random spatial sampling of the arrays makes the conventional Fourier-based imaging algorithm [8] no longer straightforwardly applicable for 3-D image formation. In practice, coherent summation methods, i.e., backprojection and Kirchhoff migration, and computational approaches are usually used to reconstruct 3-D images [7]. However, they are very computationally expensive.

So it is still in great demand to develop an efficient imaging algorithm for 3-D imaging with general planar arrays. Range migration algorithm (RMA, also known as F-K migration, phase-shift migration) is an efficient and robust approach to focus the time response of seismic wave to obtain an image of the physical geological structure. In the past decades, it has been utilized to process electromagnetic (EM) waves for the applications of, for instance, ground penetrating radar

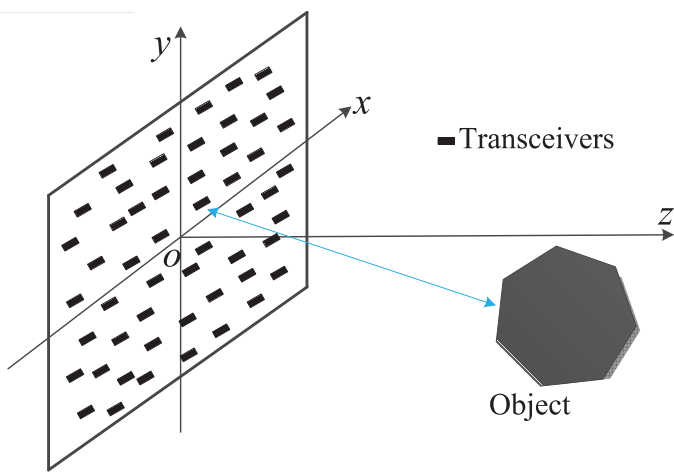

Fig. 1: Geometrical configuration for 3-D imaging with irregular planar array.

(GPR) and synthetic aperture radar (SAR). To exploit its high efficiency, the RMA has also been modified to handle the Radial-scanned Synthetic Aperture Radar (Rad-SAR) imaging by using the Nonuniform fast Fourier transform (NUFFT) [4]. However, it is mainly applicable to 3-D imaging with a planar array on a polar grid. To further explore the potential of the application of the RMA to general irregular planar arrays, we propose to use Voronoi partition to deal with the nonuniform spatial sampling and then take advantage of NUFFT for signal spectra reconstruction in the wavenumber domain. The proposed imaging algorithm is named as the NUFFT-based RMA. It not only inherits the advantage of the high efficiency of the conventional RMA but also is generally applicable to 3-D imaging with arbitrary planar arrays.

The rest of the paper is organized as follows. In section II, the formulation of 3-D imaging with irregular planar arrays is briefly described and the NUFFT-based RMA is presented. Then section III shows some numerical simulations to demonstrate the proposed imaging algorithm. Finally, some conclusions are drawn in section IV.

\section{FORMULATION FOR 3-D IMAGING WITH IRREGULAR PLANAR ARRAYS}

Assume an irregular planar array is placed on the xoy plane and radiates the EM waves towards the positive $z$ direction. The geometrical configuration is shown in Fig. 1. 
The antennas within the array aperture work in a monostatic configuration. The scattered signal acquired by an antenna at $(x, y, 0)$ is represented as $p(t, x, y, 0)$ in the time domain. For rectilinear planar arrays, the signals $p(t, x, y, 0)$ acquired by all the antennas with the aperture can be coherently and efficiently focused with the range migration algorithm, which could be summarized as the following operations [9]:

$$
\begin{aligned}
p(t, x, y, z=0) & \rightarrow P\left(f, k_{x}, k_{y}, z=0\right) \\
& \rightarrow P\left(f, k_{x}, k_{y}, z\right) \rightarrow f(t=0, x, y, z) .
\end{aligned}
$$

In particular, the operation from $p(t, x, y, z=0)$ to $P\left(f, k_{x}, k_{y}, z=0\right)$ is generally implemented by a threedimensional (3-D) fast Fourier Transform (FFT), i.e., a one-dimensional (1-D) FFT with respect to the time $t$ and a two-dimensional (2-D) FFT with respect to $x$ and $y$ over the array aperture. Then the wavefield at the measurement plane $P\left(f, k_{x}, k_{y}, z=0\right)$ is extrapolated to get the wavefield $P\left(f, k_{x}, k_{y}, z\right)$ at a particular down-range $z$. In the last step, a 3-D inverse FFT (IFFT) is taken to obtain a focused 3-D image.

However, all the FFT-related operations in the first and third steps require the signals to be uniformly sampled in the time and spatial domain. Although signals are usually uniformly sampled in time, nonuniform/random antenna arrays are frequently designed for spatial signal acquisition with the considerations of cost, coupling suppression, lower sidelobe levels, etc. As a result, they generally lead to nonrectilinear spatial sampling within the aperture. Consequently, the 3-D FFT is not straightforwardly applicable to compute the signal spectrum in the frequency-wavenumber (i.e., $f-k$ ) domain, which hinders the use of RMA for 3-D image formation with general irregular planar arrays.

To circumvent the aforementioned problem, a NUFFT-based RMA is developed by exploiting Voronoi partition to tackle the nonuniform spatial sampling in this paper. More details are presented as follows.

\section{A. Signal Spectrum Reconstruction}

The signal spectrum is typically acquired by performing 3D Fourier transform (FT) over time and 2-D space, which is analytically expressed as

$$
\begin{aligned}
& P\left(f, k_{x}, k_{y}, z=0\right) \\
& =\iint_{x, y} \int_{t} p(t, x, y, z=0) e^{-j \omega t} \mathrm{~d} t \cdot e^{-j k_{x} x-j k_{y} y} \mathrm{~d} x \mathrm{~d} y
\end{aligned}
$$

For signals uniformly sampled in time and space, (1) can be efficiently implemented by a 3-D FFT. However, for the signals acquired by irregular planar arrays, they are sampled irregularly in space. Hence, as mentioned above, the 3-D FFT is no longer applicable to transform the collected signals in the time-space (i.e., $t-x$ ) domain to their Fourier counterparts in the $f$ - $k$ domain. To compute the signal spectrum of the nonuniformly sampled signals, the integral in (1) can be replaced with the Riemann sum over the $N$ discrete sampling positions $\left[\left(x_{0}, y_{0}\right),\left(x_{1}, y_{1}\right), \cdots,\left(x_{N-1}, y_{N-1}\right)\right]$ :

$$
\begin{aligned}
& P\left(f, k_{x}, k_{y}, z=0\right) \\
& \quad=\sum_{n=0}^{N-1} P\left(f, x_{n}, y_{n}, z=0\right) e^{-j\left(k_{x} x_{n}+k_{y} y_{n}\right)} \Delta S_{n}
\end{aligned}
$$

where $P\left(f, x_{n}, y_{n}, z=0\right)=\int_{t} p\left(t, x_{n}, y_{n}, z=0\right) e^{-j \omega t} \mathrm{~d} t$ and $\Delta S_{n}$ is the area related to the sampling position $\left(x_{n}, y_{n}, 0\right)$. For uniform rectilinear spatial sampling, the area $\Delta S_{n}$ is equal for all the sampling positions. For irregular spatial sampling, we suggest employing the Voronoi partition to cope with their nonuniform distribution. By taking the Voronoi partition of the array aperture, the area of each Voronoi cell is assigned to the corresponding sample inside it. Then, (2) can be regarded as a 2-D nonuniform discrete Fourier transform (NDFT) of $P\left(f, x_{n}, y_{n}, z=0\right) \Delta S_{n}$; thus, it could be efficiently computed via the 2-D Nonuniform Fast Fourier transform (NUFFT) algorithm [10], [11].

\section{B. $k$-space Interpolation}

In the traditional RMA, a $k$-space interpolation is needed to realize the mapping from $k=2 \pi f / c$ to $k_{z}$ before taking a 3-D inverse FT to get a focused image. Explicitly, the 3-D inverse FT can be expressed as

$$
\begin{aligned}
& f(t=0, x, y, z) \\
& =\iiint P\left(f, k_{x}, k_{y}, z=0\right) e^{j k_{x} x+j k_{y} y+j k_{z} z} d k_{x} d k_{y} d \omega \\
& =c^{2} \iiint \frac{k_{z}}{4 \omega} \cdot P\left(f, k_{x}, k_{y}, z=0\right) e^{j k_{x} x+j k_{y} y+j k_{z} z} d k_{x} d k_{y} d k_{z}
\end{aligned}
$$

where $\omega=2 \pi f$ is the angular frequency, and it relates to the wavenumbers via

$$
k_{z}=\sqrt{(2 \omega / c)^{2}-k_{x}^{2}-k_{y}^{2}}
$$

As $\omega$ is uniformly sampled and $k_{x}$ and $k_{y}$ are also assumed to be uniformly sampled in the wavenumber domain, then the resultant $k_{z}$ is nonuniform based on the mapping in (4). Thus, to facilitate the application of the 3-D FFT for the image formation in (3), a mapping from $P\left(f, k_{x}, k_{y}, z=0\right)$ on a uniform grid of $f$ to $\bar{P}\left(k_{x}, k_{y}, k_{z}, z=0\right)$ on a uniform grid of $k_{z}$ is needed. Typically the Stolt interpolation is an accurate approach to implement this mapping point by point, but it is very computationally heavy. Although the Stolt interpolation can be replaced with regular interpolation approaches, such as linear, cubic, and spline interpolation, to improve the efficiency, sometimes the accuracy is not sufficient. To achieve sufficient accuracy as well as high efficiency, nonuniform fast Fourier transform (NUFFT) is introduced to substitute for the Stolt interpolation and a 1-D IFFT [9], [12]. In [13], we have extended this approach to the range migration for MIMO array imaging. 


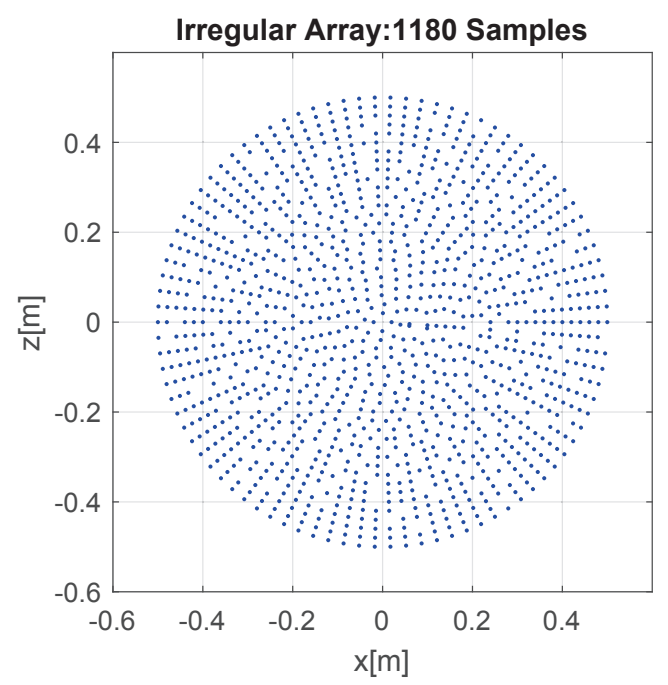

(a)

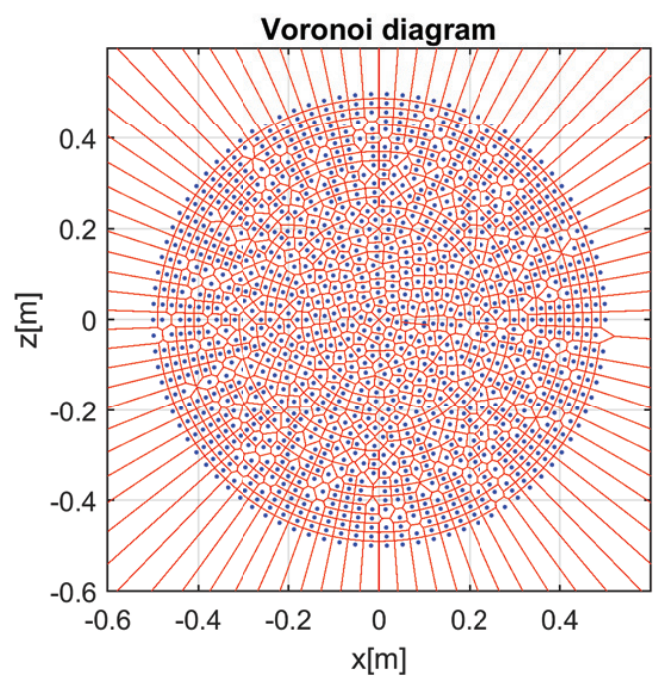

(b)

Fig. 2: The irregular planar array with 1180 spatial samples used for 3-D imaging. (a) shows the irregular array topology, and (b) the related Voronoi diagram.

\section{NUMERICAL SimUlations}

In this section, 3-D imaging simulations with irregular planar arrays are presented to demonstrate the performance of the proposed approach. An irregular circular antenna array, shown in Fig. 2(a), is positioned on the xoz plane while the $y$-axis points towards the scene of illumination and forms a right-handed coordinate system with the $x$ - and $z$-axes. The irregular array consists of 1180 transceivers (i.e., samples) within the aperture. The Hertz dipoles are used as radiators in the irregular array, and their operating frequencies sweep from 2 to $10 \mathrm{GHz}$ with steps of $100 \mathrm{MHz}$. An "E"-shaped perfect electric conductor (PEC) object is put in front of the antenna

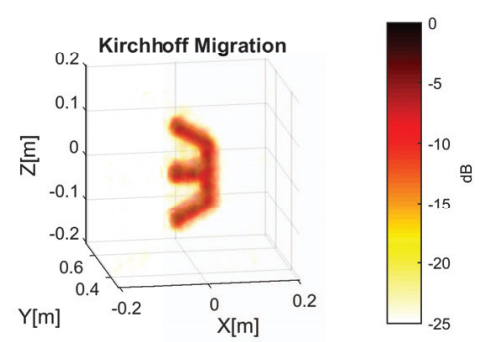

(a)

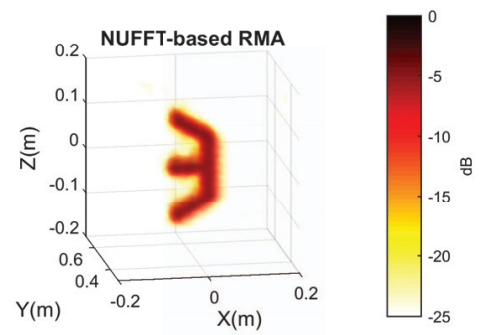

(b)

Fig. 3: The 3-D images of the signals from 6 to $8 \mathrm{GHz}$ with the irregular array focused by (a) Kirchhoff migration and (b) NUFFT-based RMA.

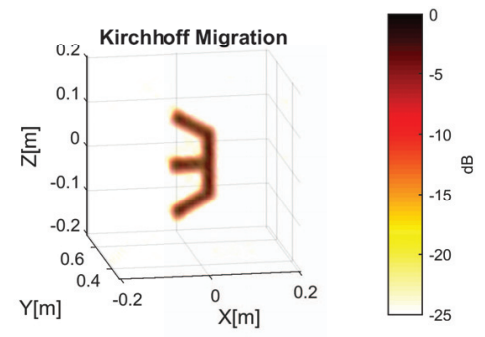

(a)

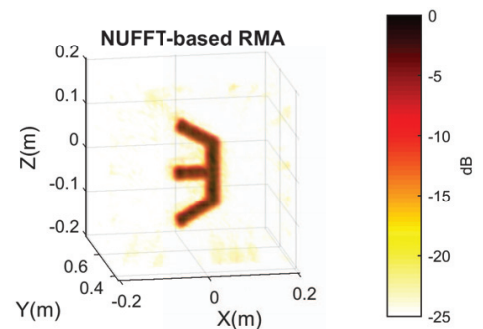

(b)

Fig. 4: The 3-D images with the irregular array focused by (a) Kirchhoff migration and (b) NUFFT-based RMA.

array at a distance of $0.5 \mathrm{~m}$. The synthetic electromagnetic (EM) signals are generated in the frequency domain by using the commercial EM software FEKO with the Method of Moments (MoM) solver.

After obtaining the synthetic EM data in the frequency domain, the proposed NUFFT-based RMA can be used for 


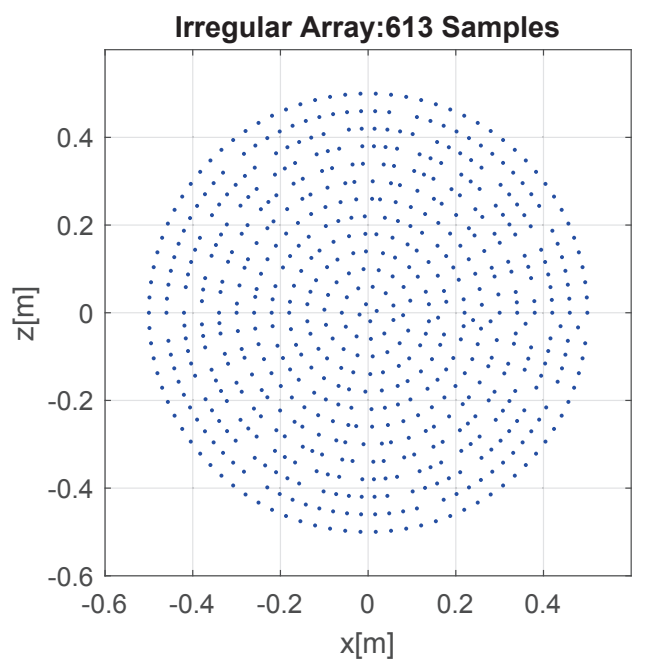

(a)

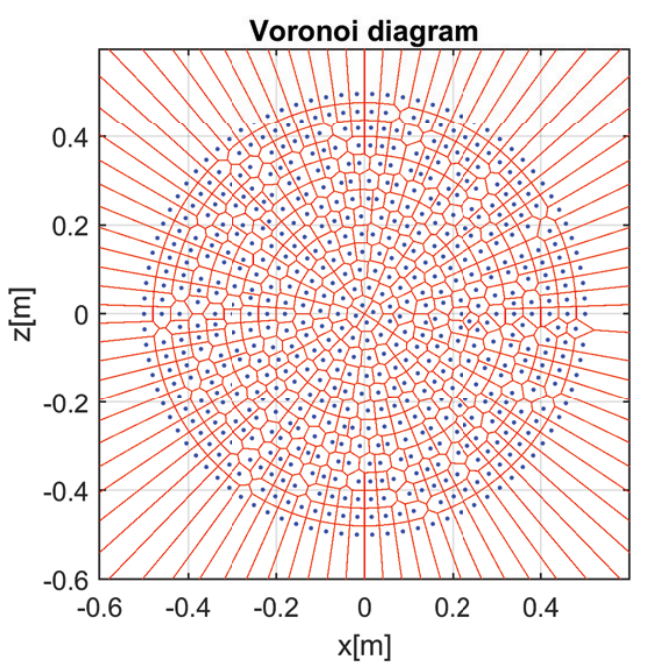

(b)

Fig. 5: The irregular planar array with 613 spatial samples. (a) shows the topology of the irregular array, and (b) the related Voronoi diagram.

image formation. Firstly, we use part of the synthetic data with frequencies from 6 to $8 \mathrm{GHz}$ for relatively narrow-band 3-D imaging. To account for the weighting effects of nonuniform spatial sampling, we first take the Voronoi partition of the array aperture [as shown in Fig. 2(b)] and then multiply the signals acquired at each spatial sampling position by the area of its corresponding Voronoi cell. The reconstructed image is shown in Fig. 3(b). Meanwhile, for comparison, the synthetic EM data with frequencies from 6 to $8 \mathrm{GHz}$ are also converted into the time domain with the FFT after applying a Hanning window. Then. the time-domain signals are focused with the Kirchhoff migration algorithm, and the obtained image is presented in Fig. 3(a). From Fig. 3(b), one can see that the proposed NUFFT-based RMA provides

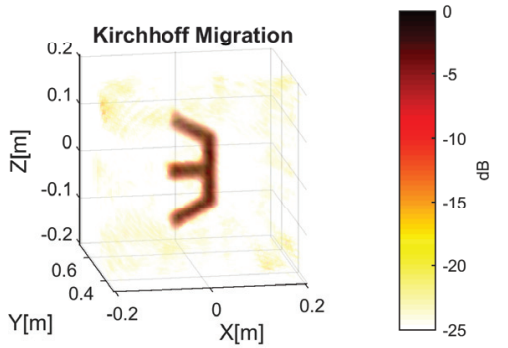

(a)

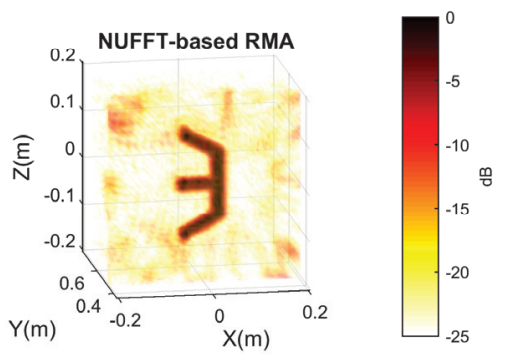

(b)

Fig. 6: The reconstructed 3-D images with the irregular array of 613 spatial samples. (a) Kirchhoff migration and (b) NUFFT-based RMA.

a well-focused image which is almost identical to Fig. 3(a). Moreover, the contrast of the target's image in Fig. 3(b) is slightly sharper than that in Fig. 3(a). The reason is that the relatively narrow bandwidth of the signal results in a pulse with a long oscillating tail; thus, after focusing operation in the time domain with Kirchhoff migration, a small part of the power spreads to the surrounding area of the object. By contrast, the NUFFT-based RMA directly focuses the signals in the $f$ - $k$ domain, which makes the power of signals more concentrated on the region of objects.

Moreover, the NUFFT-based RMA is much more efficient than the Kirchhoff migration. To facilitate the comparison, both imaging algorithms were used to reconstruct a 3-D image of the volume $[-0.496,0.496] \mathrm{m} \times[0,3] \mathrm{m} \times[-0.496,0.486] \mathrm{m}$ with sampling intervals of $7.5 \mathrm{~mm}, 1.5 \mathrm{~cm}$, and $7.5 \mathrm{~mm}$ along the $x-, y-$, and $z$-axes. They were implemented in MATLAB and ran in a computer with Intel Core i5-3470 CPU @ 3.2GHz and $8 \mathrm{~GB}$ random access memory (RAM). The simulation shows that the NUFFT-based RMA took $1.04 \mathrm{~s}$ while the Kirchhoff migration consumed $157.27 \mathrm{~s}$.

A second simulation is carried out by using all the synthetic data with frequencies from 2 to $10 \mathrm{GHz}$. Utilizing both the NUFFT-based RMA and Kirchhoff migration algorithms, the 3-D images of the "E"-shaped object are reconstructed and presented in Fig. 4. Comparing Fig. 4, one can see that both NUFFT-based RMA and Kirchhoff migration algorithms reconstruct very sharp images of the target. However, in Fig. 4(b), slightly larger artifacts that are smaller than $-20 \mathrm{~dB}$ are observed surrounding the objects in contrast to Fig. 4(a), 
which is caused by the errors/aliasing of the $f-k$ domain signal spectra reconstructed by the NUFFT, especially for the high-frequency components of the signals. In terms of the computational efficiency, the NUFFT-based RMA and Kirchhoff migration cost $1.77 \mathrm{~s}$ and $159.76 \mathrm{~s}$, respectively, to reconstruct 3-D images of the same volume as in the first simulation.

Furthermore, to investigate the effects of spatial sampling on the imaging performance of the NUFFT-based RMA, one more simulation is conducted with a sparser circular antenna array. The sparse circular has the same dimension as that used in previous simulations but contains 613 elements/samples. The array topology and the corresponding Voronoi diagram are shown in Fig. 5. The operational frequencies range from 2 to $10 \mathrm{GHz}$. The same "E"-shaped object and geometrical configuration as the previous simulations are used for synthetic data generation. After getting the synthetic EM data, the NUFFT-based RMA and Kirchhoff migration are utilized for image formation. The reconstructed images are shown in Fig. 6. In both Fig. 6(a) and (b), some artifacts are perceived in the region surrounding the object, which is caused by the sparse sampling of the antenna array. Since the sparse spatial sampling of the antenna array induces severe aliasing of the wavenumber-domain spectrum reconstructed with the NUFFT, especially for the high frequencies, it leads to larger artifacts in Fig. 6(b) compared to that in Fig. 6(a). This can be further confirmed by comparing the images in Fig. 4(b) and Fig. 6(b). Additionally, although the smaller number of spatial samples of the antenna array decreases the computational load of the Kirchhoff migration, it took 99.88 s to reconstruct a 3-D image of the same volume mentioned before but is still much less efficient than the NUFFT-based RMA that cost $1.75 \mathrm{~s}$ in this case.

\section{CONCLUSION}

A nonuniform fast Fourier transform-based range migration algorithm is proposed for 3-D imaging with irregular planar arrays. In terms of image qualities, the proposed algorithm achieves comparable performance compared to the Kirchhoff migration. However, thanks to the use of FFT and NUFFT during the imaging procedure, it is much more efficient than the Kirchhoff migration. So, the NUFFT-based RMA provides the potential for (near-) real-time high-quality imaging with irregular planar arrays.

\section{REFERENCES}

[1] B. Steinberg, Principles of aperture and array system design: including random and adaptive arrays. John Wiley \& Sons Canada, Limited, 1976.

[2] J. L. Schwartz and B. D. Steinberg, "Ultrasparse, ultrawideband arrays," Ultrasonics, Ferroelectrics and Frequency Control, IEEE Transactions on, vol. 45, no. 2, pp. 376-393, 1998.

[3] A. Austeng and S. Holm, "Sparse 2-d arrays for 3-d phased array imaging - design methods," Ultrasonics, Ferroelectrics, and Frequency Control, IEEE Transactions on, vol. 49, no. 8, pp. 1073-1086, 2002.

[4] Z. Li, J. Wang, J. Wu, and Q. H. Liu, "A fast radial scanned nearfield 3-D SAR imaging system and the reconstruction method," IEEE Transactions on Geoscience and Remote Sensing, vol. 53, no. 3, pp. 1355-1363, March 2015.
[5] J. Wang, P. Aubry, and A. Yarovoy, "A novel approach to fullpolarimetric short-range imaging with copolarized data," IEEE Transactions on Antennas and Propagation, vol. 64, no. 11, pp. 4733-4744, Nov 2016.

[6] — "A novel rotated antenna array topology for near-field 3-D fully polarimetric imaging," IEEE Transactions on Antennas and Propagation, vol. PP, no. 99, pp. 1-1, 2018.

[7] B. M. Lucotte, B. Grafulla-Gonzlez, and A. R. Harvey, "Array rotation aperture synthesis for short-range imaging at millimeter wavelengths," Radio Science, vol. 44, no. 1, pp. n/a-n/a, 2009.

[8] D. M. Sheen, D. L. McMakin, and T. E. Hall, "Three-dimensional millimeter-wave imaging for concealed weapon detection," Microwave Theory and Techniques, IEEE Transactions on, vol. 49, no. 9, pp. 1581$1592,2001$.

[9] J. Song, Q.-H. Liu, P. Torrione, and L. Collins, "Two-dimensional and three-dimensional nufft migration method for landmine detection using ground-penetrating radar," IEEE Trans. Geosci. Remote Sens., vol. 44, no. 6, pp. 1462-1469, 2006.

[10] A. Dutt and V. Rokhlin, "Fast Fourier transforms for nonequispaced data," SIAM Journal on Scientific Computing, vol. 14, no. 6, pp. 13681393, 1993.

[11] L. Greengard and J.-Y. Lee, "Accelerating the nonuniform fast fourier transform," SIAM Review, vol. 46, no. 3, pp. 443-454, 2004.

[12] B. Subiza, E. Gimeno-Nieves, J. M. Lopez-Sanchez, and J. FortunyGuasch, "An approach to SAR imaging by means of non-uniform FFTs," in Geoscience and Remote Sensing Symposium, 2003. IGARSS '03. Proceedings. 2003 IEEE International, vol. 6, pp. 4089-4091 vol.6.

[13] J. Wang, H. Cetinkaya, and A. Yarovoy, "NUFFT based frequencywavenumber domain focusing under MIMO array configurations," in Radar Conference, 2014 IEEE, pp. 1-5. 